Interim Report on SNP analysis and forensic microarray probe design for South American hemorrhagic fever viruses, tick-borne encephalitis virus, henipaviruses, Old World Arenaviruses, filoviruses, Crimean-Congo hemorrhagic fever viruses, Rift Valley fever viruses and Japanese encephalitis viruses

C. Jaing, S. Gardner

June 12, 2012 
This document was prepared as an account of work sponsored by an agency of the United States government. Neither the United States government nor Lawrence Livermore National Security, LLC, nor any of their employees makes any warranty, expressed or implied, or assumes any legal liability or responsibility for the accuracy, completeness, or usefulness of any information, apparatus, product, or process disclosed, or represents that its use would not infringe privately owned rights. Reference herein to any specific commercial product, process, or service by trade name, trademark, manufacturer, or otherwise does not necessarily constitute or imply its endorsement, recommendation, or favoring by the United States government or Lawrence Livermore National Security, LLC. The views and opinions of authors expressed herein do not necessarily state or reflect those of the United States government or Lawrence Livermore National Security, LLC, and shall not be used for advertising or product endorsement purposes.

This work performed under the auspices of the U.S. Department of Energy by Lawrence Livermore National Laboratory under Contract DE-AC52-07NA27344. 


\title{
Interim Report on SNP analysis and forensic microarray probe design for South American hemorrhagic fever viruses, tick-borne encephalitis viruses, henipaviruses, Old World Arenaviruses, filoviruses, Crimean-Congo hemorrhagic fever viruses, Rift Valley fever viruses and Japanese encephalitis viruses
}

\author{
Project Title: Forensic TaqMan and Microarray Assays for Viral Genotyping
}

\author{
Contributors
}

Shea Gardner and Crystal Jaing

Lawrence Livermore National Laboratory (LLNL), Livermore, CA

\author{
Principal Investigator and Correspondent \\ Crystal Jaing \\ 925-424-6574, jaing2@1lnl.gov
}

LLNL-TR-560677

June 5, 2012

This document was prepared as an account of work sponsored by an agency of the United States government. Neither the United States government nor Lawrence Livermore National Security, LLC, nor any of their employees makes any warranty, expressed or implied, or assumes any legal liability or responsibility for the accuracy, completeness, or usefulness of any information, apparatus, product, or process disclosed, or represents that its use would not infringe privately owned rights. Reference herein to any specific commercial product, process, or service by trade name, trademark, manufacturer, or otherwise does not necessarily constitute or imply its endorsement, recommendation, or favoring by the United States government or Lawrence Livermore National Security, LLC. The views and opinions of authors expressed herein do not necessarily state or reflect those of the United States government or Lawrence Livermore National Security, LLC, and shall not be used for advertising or product endorsement purposes.

This work performed under the auspices of the U.S. Department of Energy by Lawrence Livermore National Laboratory under Contract DE-AC52-07NA27344. 


\section{Introduction}

The goal of this project is to develop forensic genotyping assays for select agent viruses, enhancing the current capabilities for the viral bioforensics and law enforcement community. We used a multipronged approach combining bioinformatics analysis, PCR-enriched samples, microarrays and TaqMan assays to develop high resolution and cost effective genotyping methods for strain level forensic discrimination of viruses. We have leveraged substantial experience and efficiency gained through year 1 on software development, SNP discovery, TaqMan signature design and phylogenetic signature mapping to scale up the development of forensics signatures in year 2. In this report, we have summarized the whole genome wide SNP analysis and microarray probe design for forensics characterization of South American hemorrhagic fever viruses, tickborne encephalitis viruses and henipaviruses, Old World Arenaviruses, filoviruses, Crimean-Congo hemorrhagic fever virus, Rift Valley fever virus and Japanese encephalitis virus.

\section{Methods}

SNP analyses were performed on all available full genomes or segments using kSNP. kSNP is a bioinformatics tool for sequence comparison and can scale to hundreds of bacterial or viral genomes, and can be used for finished and/or draft genomes available as unassembled contigs [1]. The method is fast to compute, finding SNPs and building a SNP phylogeny in seconds to hours depending on input. The approach can handle as input hundreds of megabases to gigabases of sequence in a single run. $\mathrm{k}=13$ identified more SNP alleles than $\mathrm{k}=25$. Fewer SNPs were found with the larger $\mathrm{k}$ because a longer length of conserved sequence surrounding the SNP is required. With these divergent viruses, shorter k means that SNPs in closer proximity to one another can be found, thus reducing the stringency for conservation surrounding a SNP. A value of $k=13$ for viruses should provide better resolution of unsequenced novel isolates than $\mathrm{k}=25$, so all results reported below are for $\mathrm{k}=13$, unless otherwise indicated. The results are summarized in Table 1.

Phylogenetic trees were created using:

1) Multiple sequence alignments (MSA), or full genome multiple sequence alignment with MUSCLE (http://www.drive5.com/muscle/; [2]) followed by maximum likelihood with 1000 bootstrap replicates using RAxML (http://icwww.epfl.ch/ stamatak/indexDateien/software/RAxML-Manual.7.0.4.pdf ; [3])

2) SNP Hamming distance, calculated as the number of SNPs that differ between each pair of genomes and trees built using the neighbor method in PHYLIP

3) SNP RAxML, analyzing just the SNP alleles concatentated into a string for each genome, to make a SNP alignment from multiple genomes that can be treated as though it were an MSA, then using RAxML to create a maximum likelihood tree with 1000 bootstrap replicates from this SNP alignment.

Branch specific SNP allele counts are plotted on nodes, strain/genome specific allele counts are given in brackets after strain name. For each target set, the tree (full genome MSA, SNP distance, or SNP RAxML) that results in the most SNPs mapping to the tree is shown (that is, the tree that yields the fewest homoplastic SNPs).

Microarray probes were designed for every SNP. Probe design strategy maximized sensitivity and specificity based on extensive prior lab testing on a NimbleGen microarray platform, where we demonstrated 100\% SNP allele call rates and 99.5\% accuracy (in prep, and unpublished 
reports for DHS). We determined that maximum sensitivity and SNP discrimination accuracy result

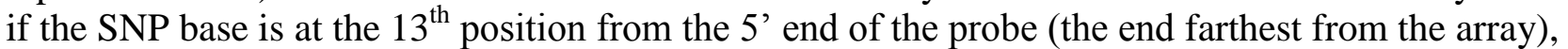
probes are between 32 and 40 bases long, and length varies so as to equalize hybridization free energy $(\Delta \mathrm{G})$ to the extent possible within the allowable length range. Probes shorter than 32 bases have high false negative rates, and longer probes are inefficient at discriminating single base mismatches. We found that $\Delta \mathrm{G}$ is a better predictor of hybridization than $\mathrm{T}_{\mathrm{m}}$. Probe candidates with hybridization free energy below $\Delta \mathrm{G}=-43 \mathrm{kcal} / \mathrm{mol}$ were shortened until either their $\Delta \mathrm{G}$ exceeded $43 \mathrm{kcal} / \mathrm{mol}$ or they reached the minimum 32 bases. Probes were designed around the SNP on both the plus and minus strands, for all 4 possible SNP alleles, and all surrounding sequence variants. We design probes for both the plus and minus strand; these are not the reverse complements of one another because the SNP does not lie at the center of the probe. There are probes for each of the 4 variants on each strand, so at least 8 probes per SNP locus. In addition, any sequence variation outside of the k-mer SNP context of conserved bases is captured in multiple alternative probes for that allele, so there may be more than 8 probes per SNP locus, although for a given hybridization, only the probe variant with the best signal is used for assessing the SNP allele at the $13^{\text {th }}$ position. Finally, probes are trimmed from the 3' end to remove any N's or other degenerate bases, and omitted altogether if doing so results in a probe less than 32 bases. If a probe is a subsequence of any other, only the shorter of the two is kept. If necessary to fit on the desired array format, probes can be omitted for alleles not represented in the target sequences, e.g. for biallelic SNPs half the probes can be pruned. Both full unpruned sets of probes and those reduced to the observed allele variants are provided.

\section{Results}

The viruses analyzed have between 5 and 144 full length finished and draft genomes or segments available for analysis (Table 1). We found between 182 and 10,276 SNP loci for each organism. The detailed phylogenetic trees are shown after Table 1. Branch-specific SNP allele counts are plotted on nodes, strain/genome specific allele counts are given in brackets after strain name. For each target set, the tree that results in the most SNPs mapping to the tree is shown (that is, the tree that yields the fewest homoplastic SNPs). 
Table 1. SNP analysis results summary.

$\begin{array}{llllll}\text { Organism } & \begin{array}{l}\text { \# target } \\ \text { sequenc } \\ \text { es }\end{array} & \begin{array}{l}\text { \# SNP } \\ \text { loci }\end{array} & \text { \# probes } & \begin{array}{l}\text { \# probes for } \\ \text { observed } \\ \text { alleles only }\end{array} & \text { Strain resolution } \\ \text { Ebola } & 22 & 3083 & 56,113 & 16,163 & \text { All strains can be uniquely resolved } \\ \text { Marburg } & 31 & 2922 & 65,413 & 18,951 & \text { All strains can be uniquely resolved } \\ \text { CCHF L } & 31 & 7216 & 233,005 & 62,837 & \text { All strains can be uniquely resolved } \\ \text { CCHF M } & 49 & 4720 & 144,741 & 38,729 & \text { All strains can be uniquely resolved } \\ \text { CCHF S } & 56 & 1073 & 40,437 & 11,145 & \text { All strains can be uniquely resolved } \\ \text { RVF L } & 62 & 1190 & 39,213 & 12,181 & \text { All strains can be uniquely resolved } \\ \text { RVF M } & 69 & 897 & 33,025 & 9,888 & \text { All strains can be uniquely resolved } \\ \text { RVF S } & 89 & 436 & 17,993 & 5,381 & \begin{array}{l}\text { Only 2 strains cannot be uniquely resolved on the } \\ \text { basis of SNPs: }\end{array}\end{array}$

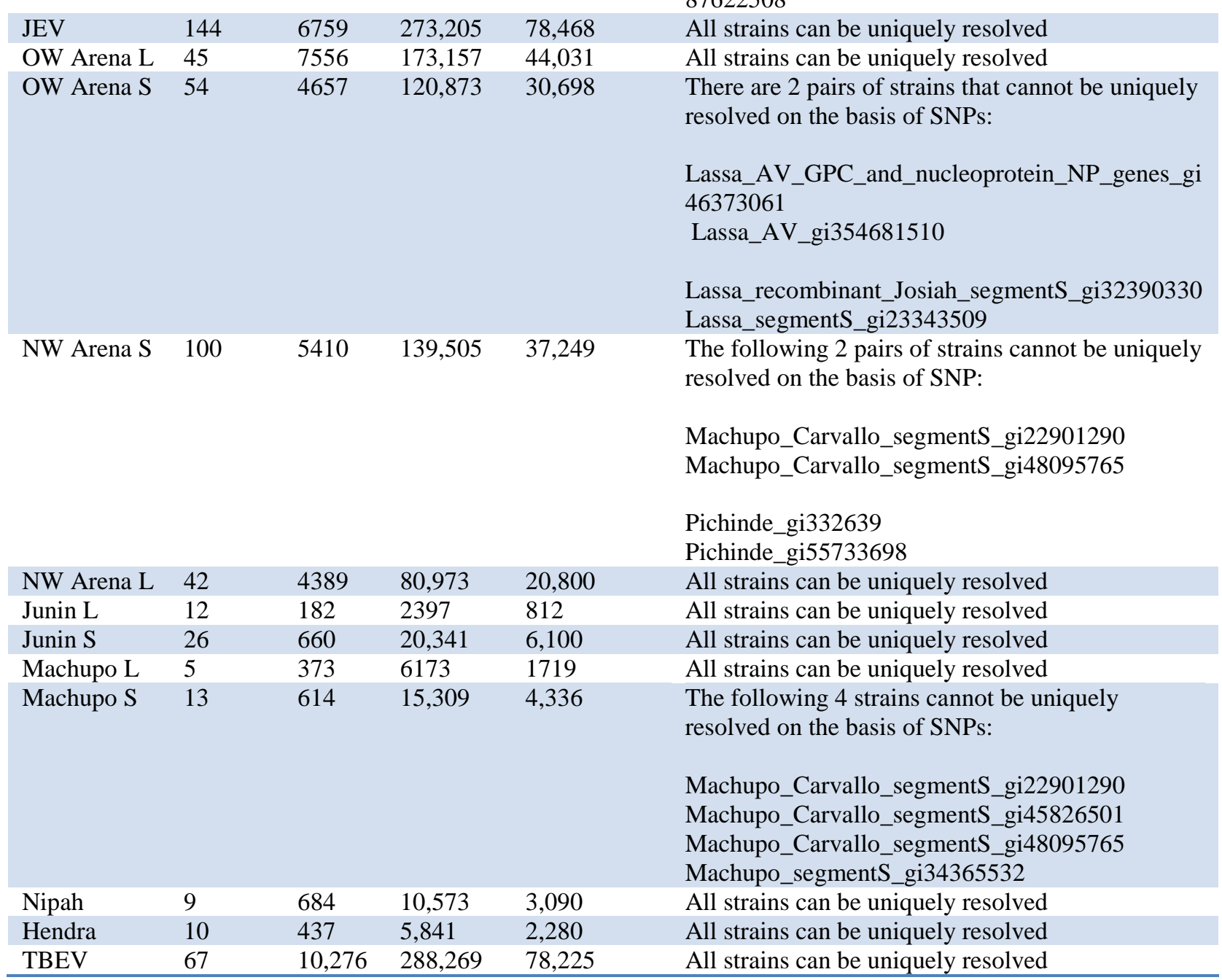

Rift_Valley_fever_200803166_segmentS_gi33042 2537

Rift_Valley_fever_Kenya_9800523_segmentS_gi 87622508

Abbreviations: $\mathrm{CCHF}=\mathrm{Crimean}-$ Congo hemorrhagic fever, $\mathrm{RVF}=$ Rift Valley fever, JEV=Japanese encephalitis virus, NW Arena=New World Arenavirus, OW Arena=Old World Arenavirus, TBEV=tick-borne encephalitis virus. 


\section{Ebola}

Number_SNPs: 3083

Number_Homoplastic_SNPs from MSA tree (shown below): 97

Number_Homoplastic_SNPs from SNP Hamming distance tree: 123

Number_Homoplastic_SNPs from SNP RAxML tree: 306

All strains can be uniquely resolved on the basis of SNPs.

Number of SNP microarray probes:

56,113 probes for all alleles

16,163 probes for observed alleles only

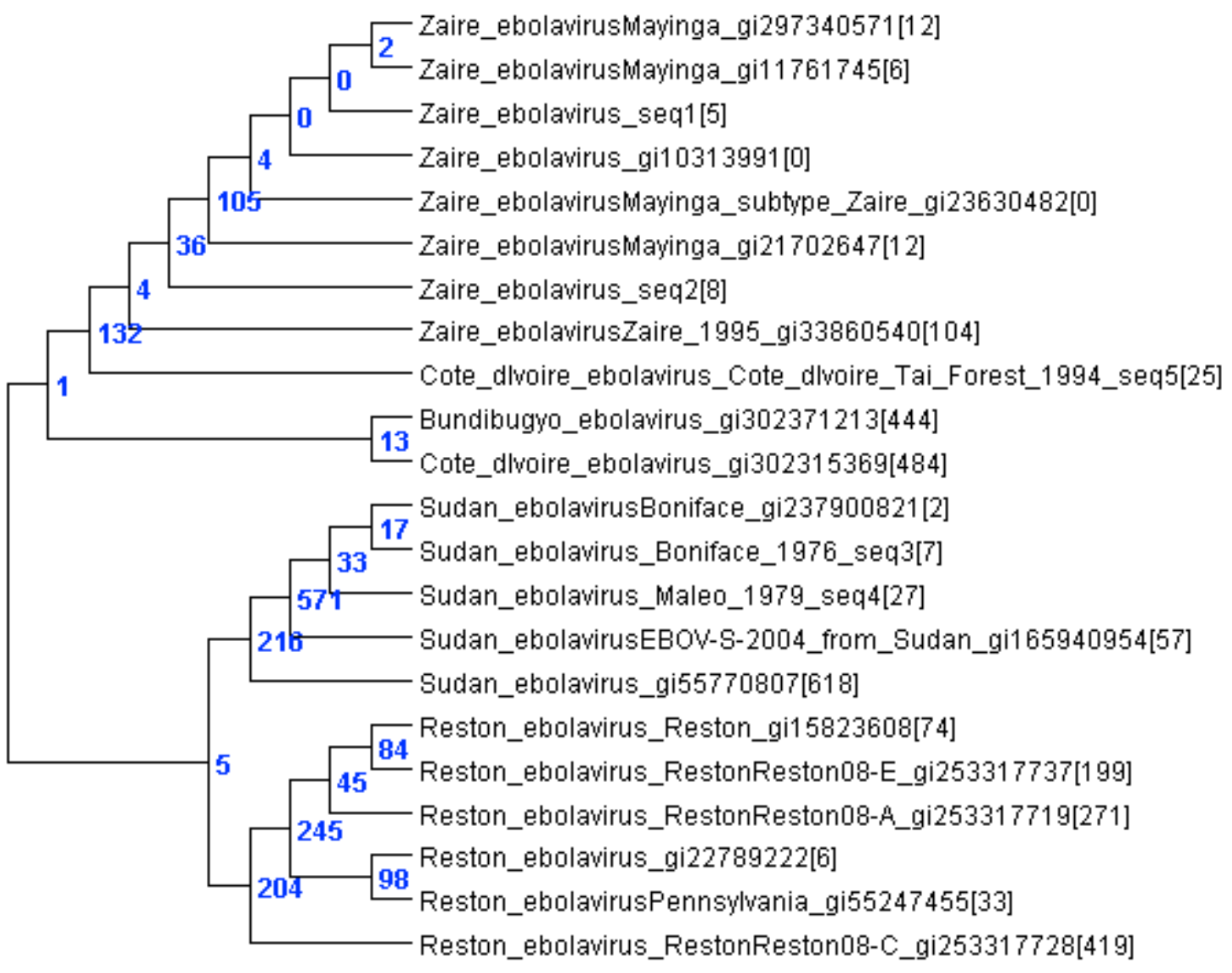




\section{Marburg}

Number_SNPs: 2922

Number_Homoplastic_SNPs from MSA tree: 1168

Number_Homoplastic_SNPs from SNP Hamming distance tree (shown below): 200

Number_Homoplastic_SNPs from SNP RAxML tree: 214

All strains can be uniquely resolved on the basis of SNPs.

Number of SNP microarray probes:

65,413 probes for all alleles

18,951 probes for observed alleles only

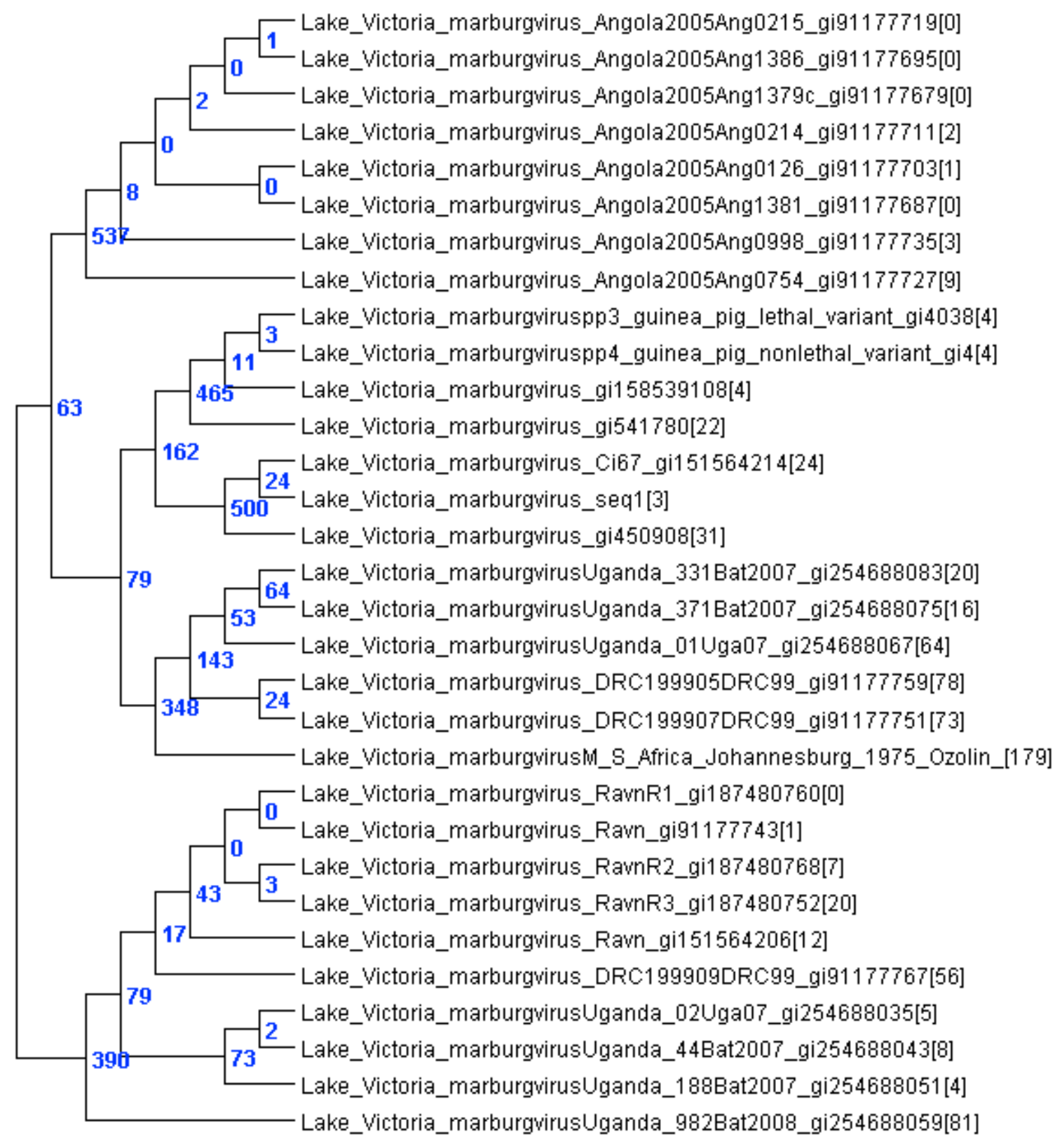




\section{CCHF L segment}

Number_SNPs: 7216

Number_Homoplastic_SNPs from MSA tree: 1422

Number_Homoplastic_SNPs from SNP Hamming distance tree (shown below): 1223

Number_Homoplastic_SNPs from SNP RAxML tree: 1270

All strains can be uniquely resolved on the basis of SNPs.

Number of SNP microarray probes:

233,005 probes for all alleles

62,837 probes for observed alleles only

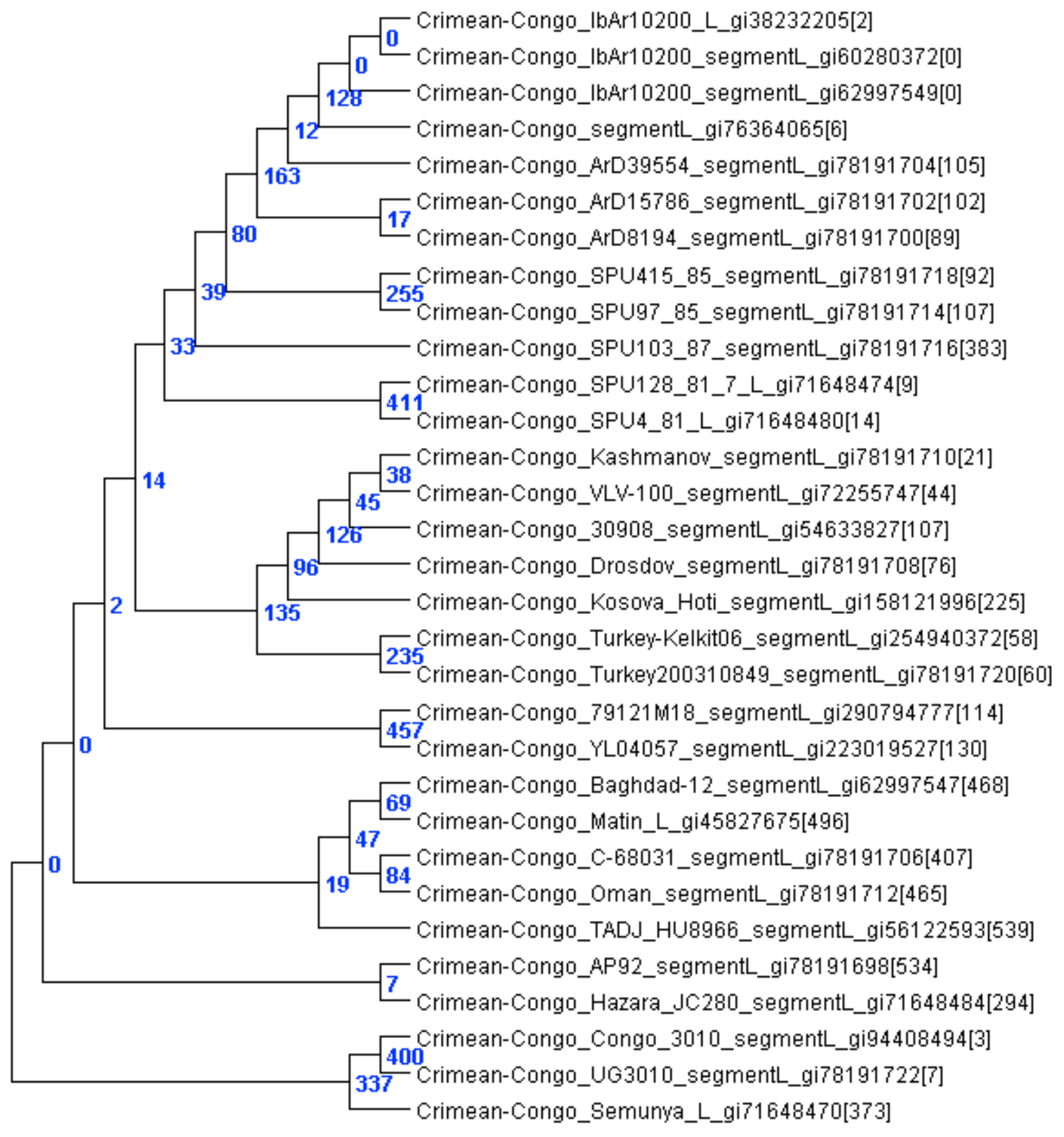




\section{CCHF M segment}

Number_SNPs: 4720

Number_Homoplastic_SNPs from MSA tree: 680

Number_Homoplastic_SNPs from SNP Hamming distance tree (shown below): 585

Number_Homoplastic_SNPs from SNP RAxML tree: 791

All strains can be uniquely resolved on the basis of SNPs.

Number of SNP microarray probes:

144,741 probes for all alleles

38,729 probes for observed alleles only

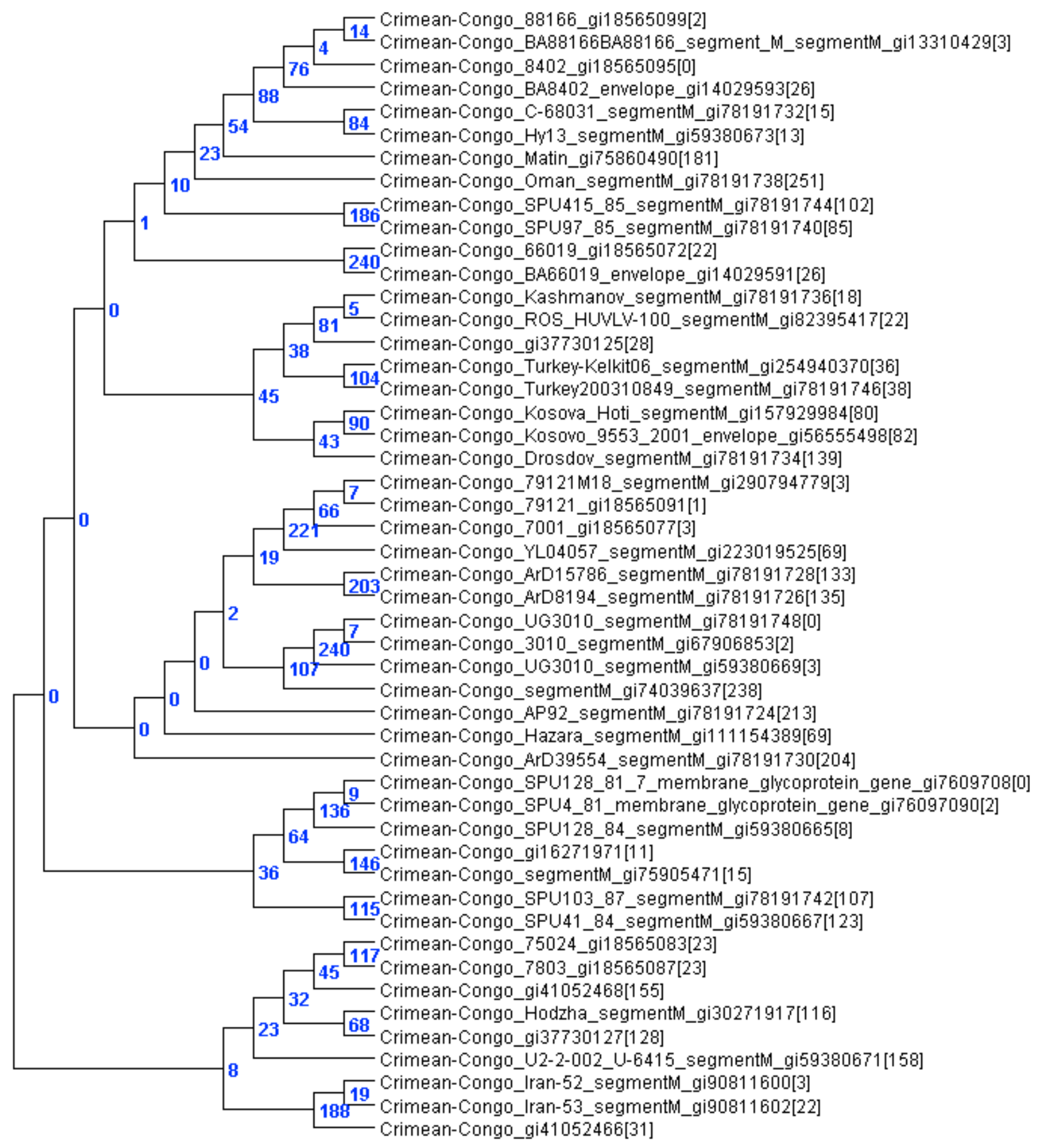




\section{CCHF S segment}

Number_SNPs: 1073

Number_Homoplastic_SNPs from MSA tree: 294

Number_Homoplastic_SNPs from SNP Hamming distance tree (shown below): 266

Number_Homoplastic_SNPs from SNP RAxML tree: 326

All strains can be uniquely resolved on the basis of SNPs.

Number of SNP microarray probes:

40,437 probes for all alleles

\section{1,145 probes for observed alleles only}

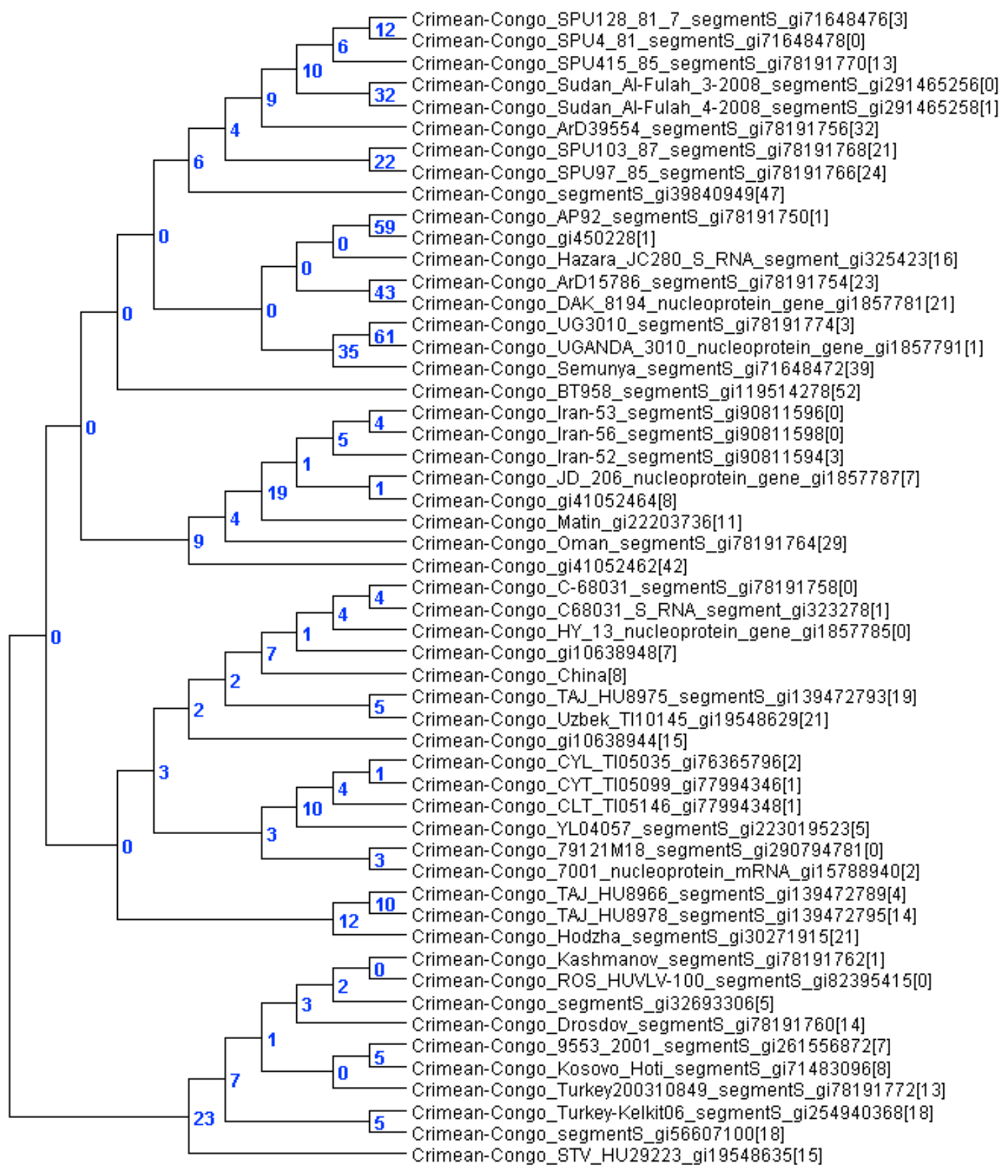




\section{RVF L segment}

Number_SNPs: 1190

Number_Homoplastic_SNPs from MSA tree (shown below): 271

Number_Homoplastic_SNPs from SNP Hamming distance tree: 276

Number_Homoplastic_SNPs from SNP RAxML tree: 271

All strains can be uniquely resolved on the basis of SNPs.

Number of SNP microarray probes:

39,213 probes for all alleles

\section{2,181 probes for observed alleles only}

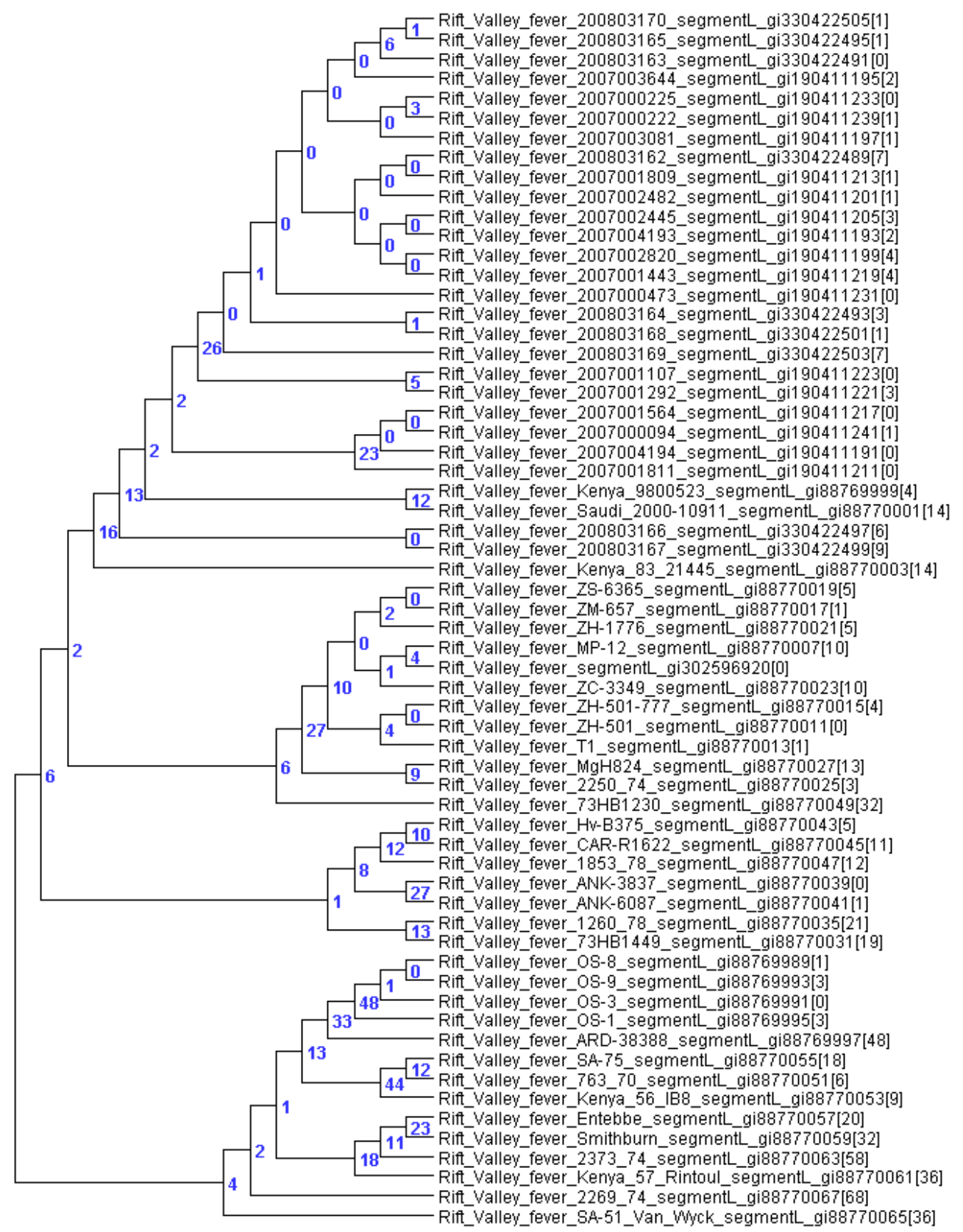




\section{RVF M segment}

Number_SNPs: 897

Number_Homoplastic_SNPs from MSA tree: 192

Number_Homoplastic_SNPs from SNP Hamming distance tree (shown below): 189

Number_Homoplastic_SNPs from SNP RAxML tree: 191

All strains can be uniquely resolved on the basis of SNPs.

Number of SNP microarray probes:

33,025 probes for all alleles

9,888 probes for observed alleles only

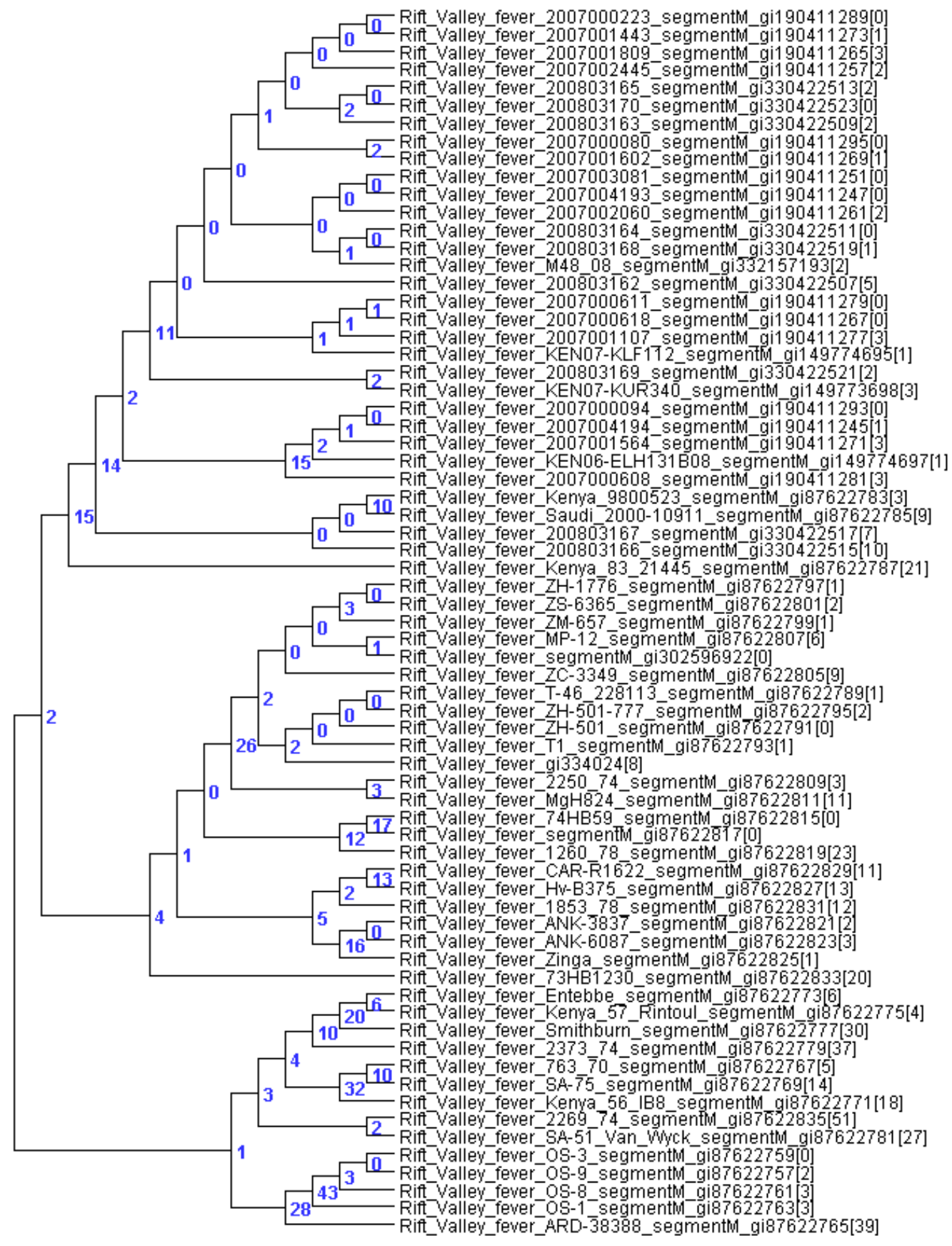




\section{RVF S segment}

Number_SNPs: 436

Number_Homoplastic_SNPs from MSA tree: 107

Number_Homoplastic_SNPs from SNP Hamming distance tree: 110

Number_Homoplastic_SNPs from SNP RAxML tree (shown below): 106

Only 2 strains cannot be uniquely resolved on the basis of SNPs:

Rift_Valley_fever_200803166_segmentS_gi330422537

Rift_Valley_fever_Kenya_9800523_segmentS_gi87622508

Number of SNP microarray probes:

17,993 probes for all alleles

5,381 probes for observed alleles only

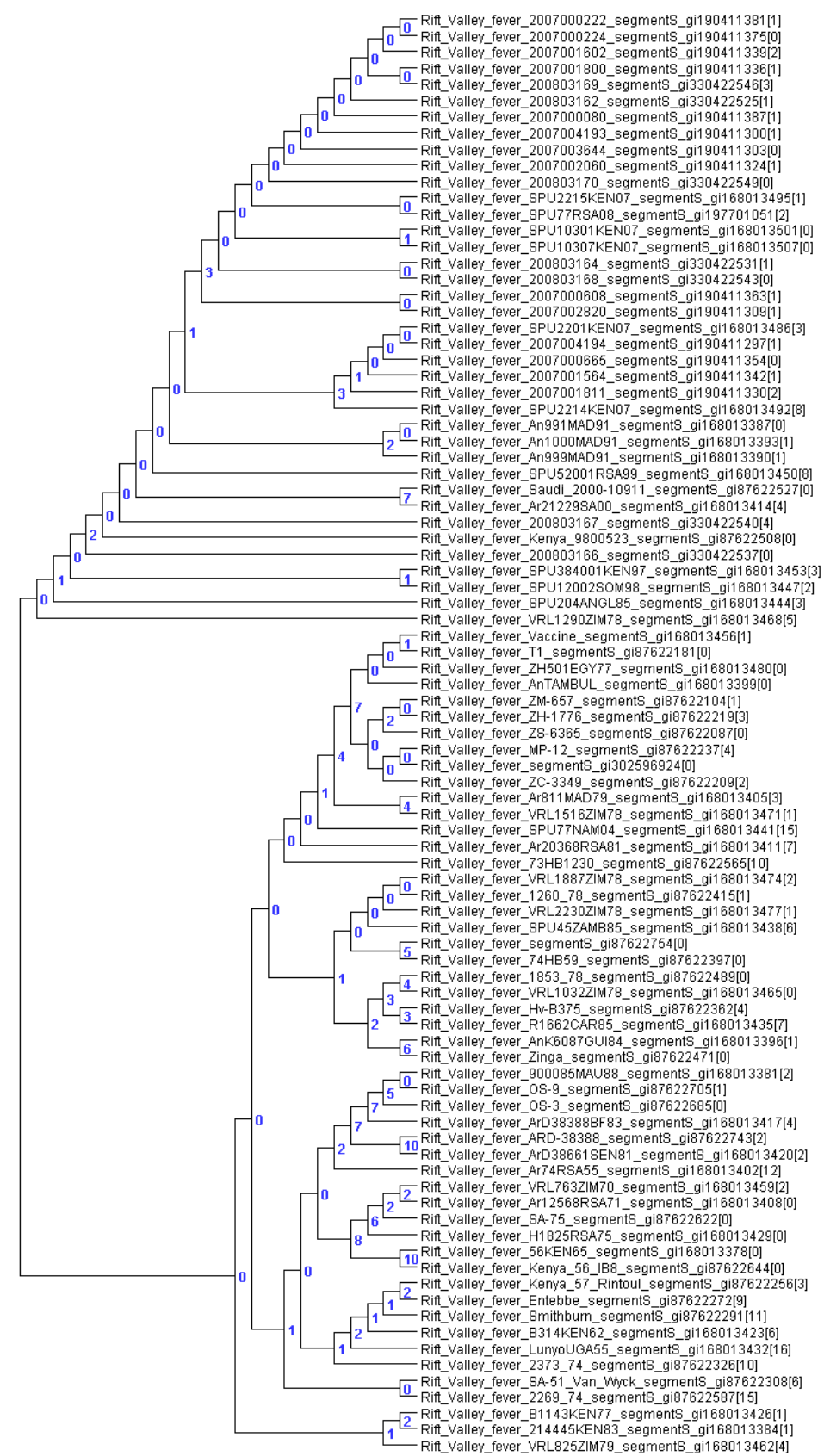




\section{JEV}

Number_SNPs: 6759

Number_Homoplastic_SNPs from MSA tree: 2050

Number_Homoplastic_SNPs from SNP Hamming distance tree (shown below): 2018

Number_Homoplastic_SNPs from SNP RAxML tree: 2139

All strains can be uniquely resolved on the basis of SNPs.

Number of SNP microarray probes:

273,205 probes for all alleles

78,468 probes for observed alleles only 


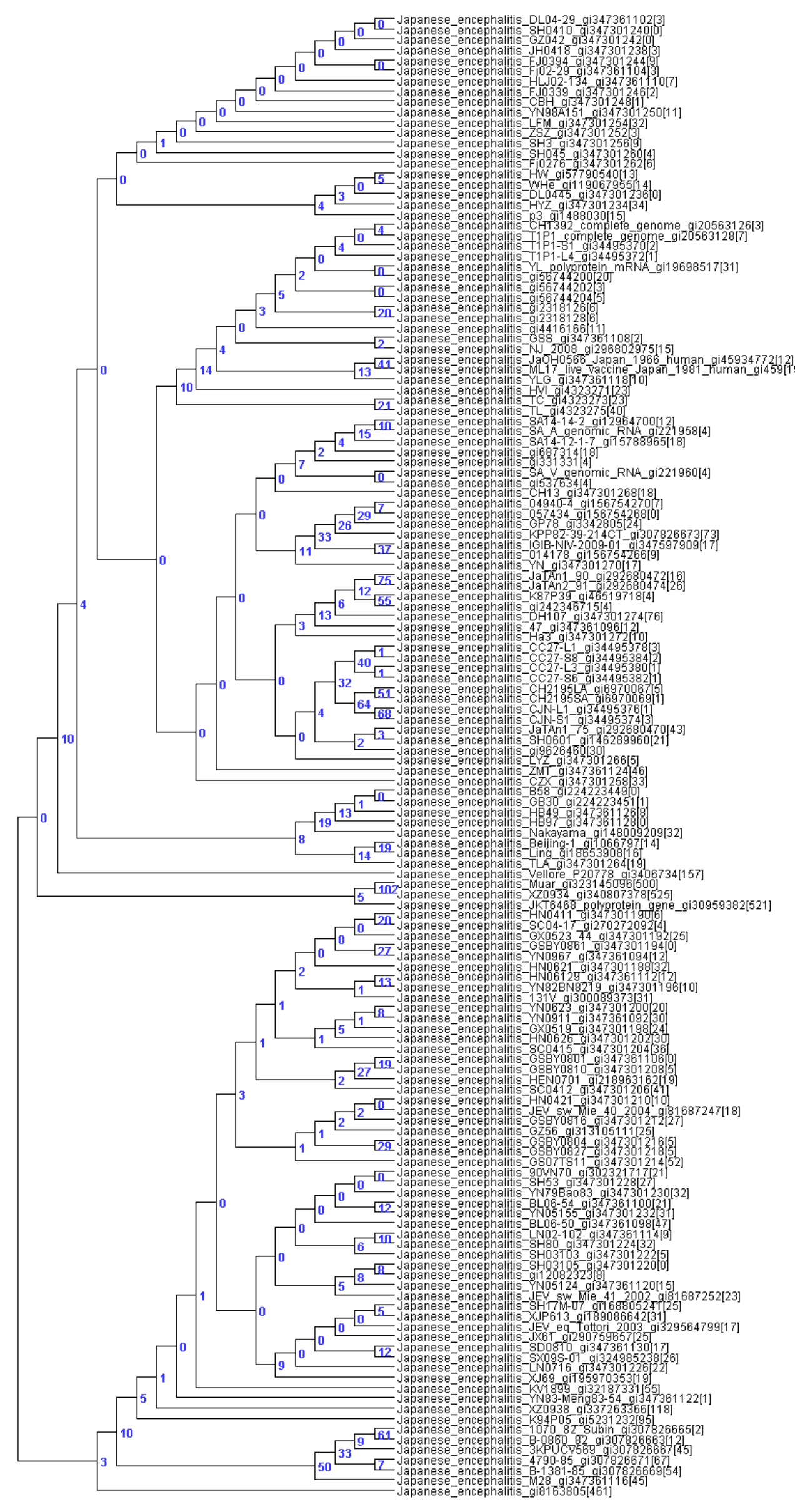




\section{Old World Arenaviruses L segment}

Number_SNPs: 7556

Number_Homoplastic_SNPs from MSA tree: 698

Number_Homoplastic_SNPs from SNP Hamming distance tree (shown below): 417

Number_Homoplastic_SNPs from SNP RAxML tree: 606

All strains can be uniquely resolved on the basis of SNPs.

Number of SNP microarray probes:

173,157 probes for all alleles

44,031 probes for observed alleles only

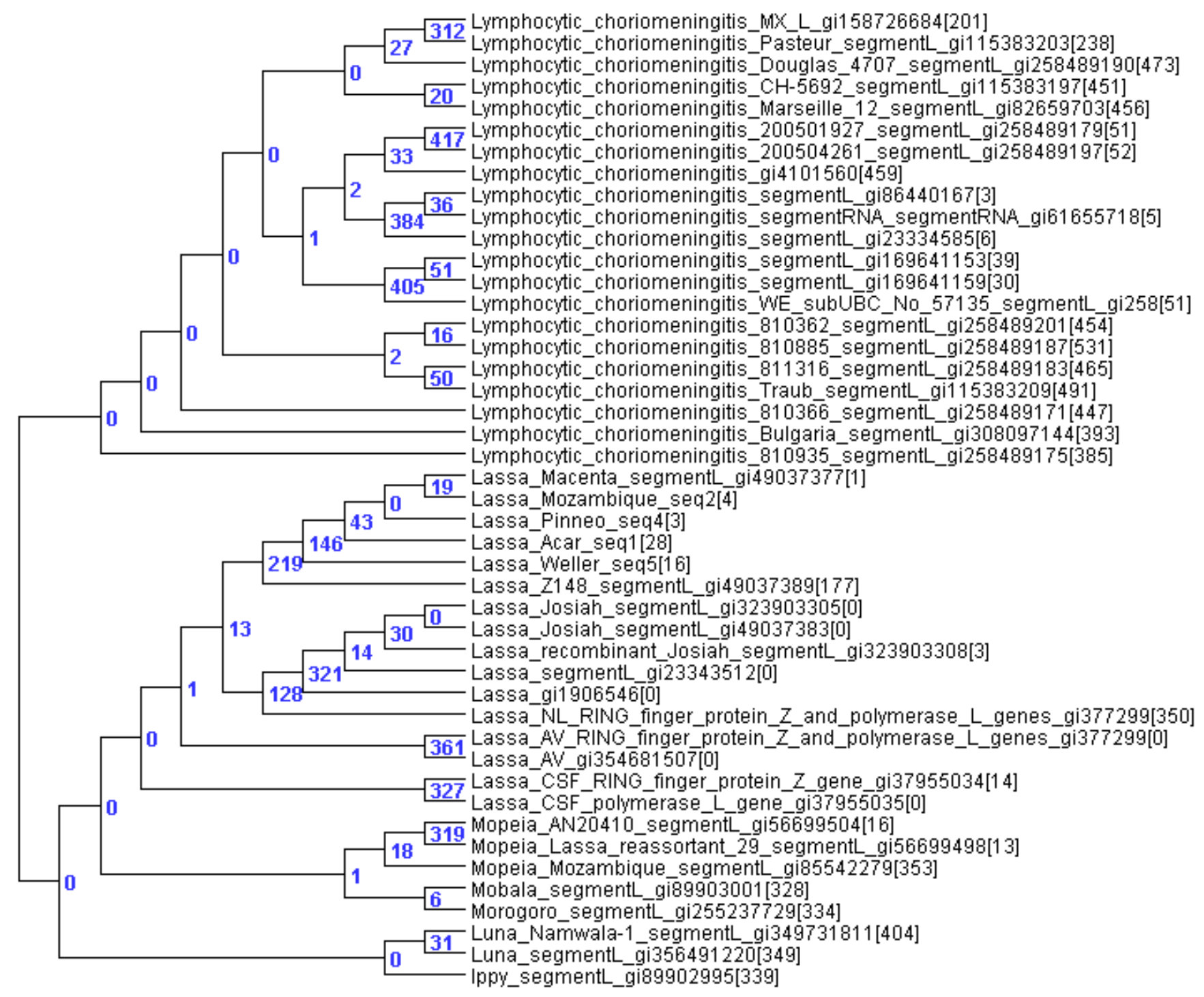




\section{Old World Arenaviruses S segment}

Number_SNPs: 4657

Number_Homoplastic_SNPs from MSA tree: 602

Number_Homoplastic_SNPs from SNP Hamming distance tree (shown below): 484

Number_Homoplastic_SNPs from SNP RAxML tree: 576

There are 2 pairs of strains that cannot be uniquely resolved on the basis of SNPs:

1 Lassa_AV_GPC_and_nucleoprotein_NP_genes_gi46373061

1 Lassa_AV_gi354681510

2 Lassa_recombinant_Josiah_segmentS_gi323903302

2 Lassa_segmentS_gi23343509

Number of SNP microarray probes:

120,873 probes for all alleles

30,698 probes for observed alleles only

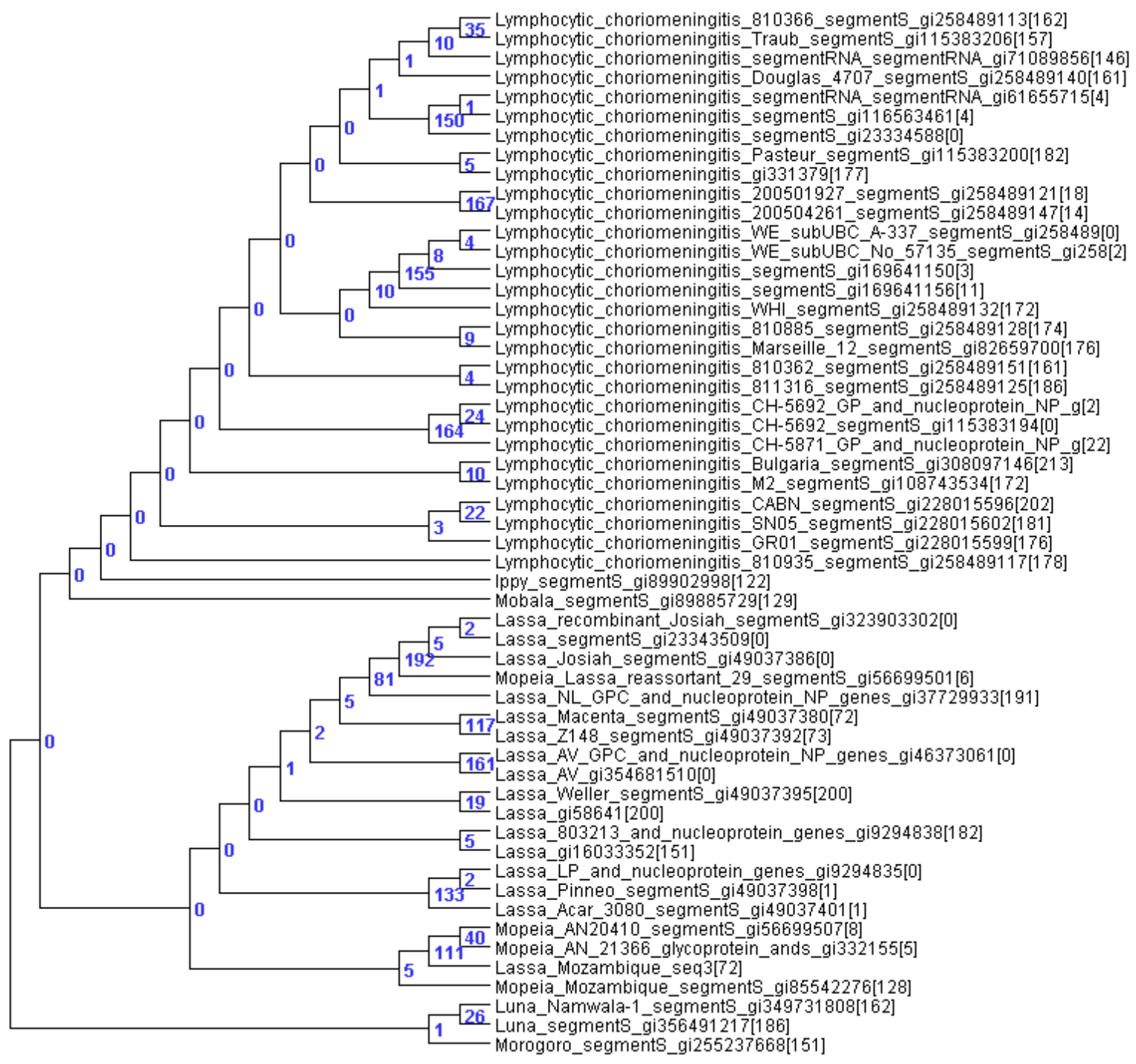


Junin L segment

Number_SNPs: 182

Number_Homoplastic_SNPs from MSA tree: 7

Number_Homoplastic_SNPs from SNP Hamming distance tree: 5

Number_Homoplastic_SNPs from SNP RAxML tree (shown below): 4 All strains can be uniquely resolved on the basis of SNPs.

Number of SNP microarray probes:

2397 probes for all alleles

812 probes for observed alleles only

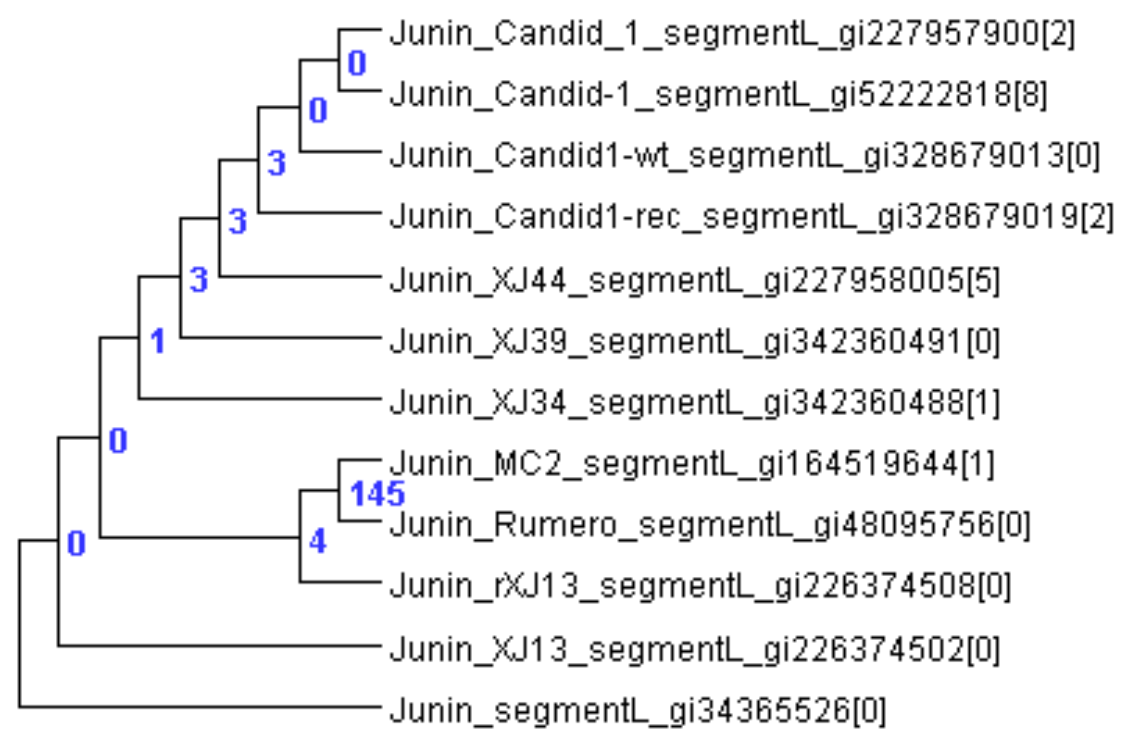


Junin S segment

Number_SNPs: 660

Number_Homoplastic_SNPs from MSA tree: 162

Number_Homoplastic_SNPs from SNP Hamming distance tree: 161

Number_Homoplastic_SNPs from SNP RAxML tree (shown below): 160

All strains can be uniquely resolved on the basis of SNPs.

Number of SNP microarray probes:

20,341 probes for all alleles

6,100 probes for observed alleles only

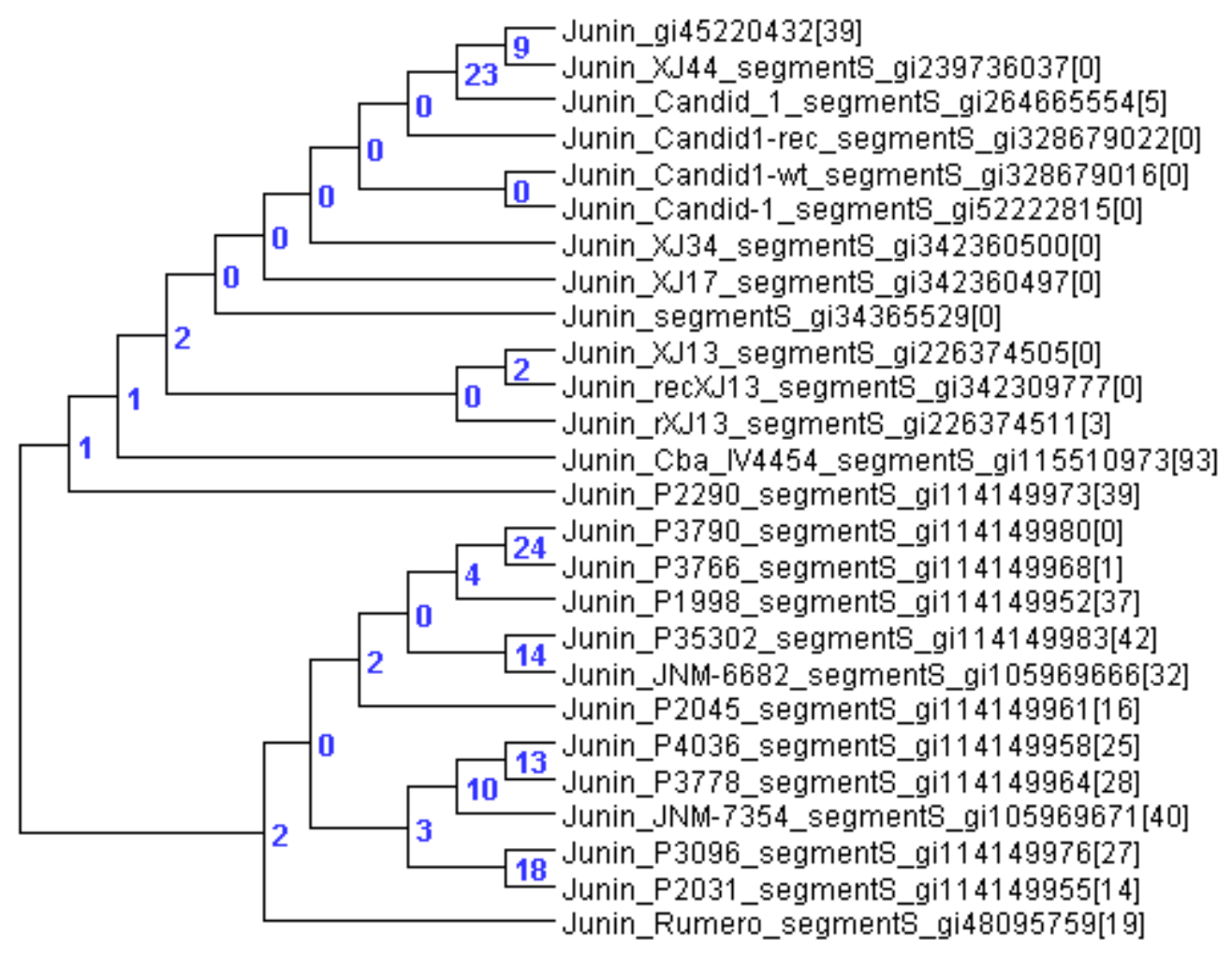


Machupo L segment

Number_SNPs: 373

Number_Homoplastic_SNPs from MSA tree (shown below): 0

Number_Homoplastic_SNPs from SNP Hamming distance tree: 0

Number_Homoplastic_SNPs from SNP RAxML tree: 0

All strains can be uniquely resolved on the basis of SNPs.

Number of SNP microarray probes:

6173 probes for all alleles

1719 probes for observed alleles only

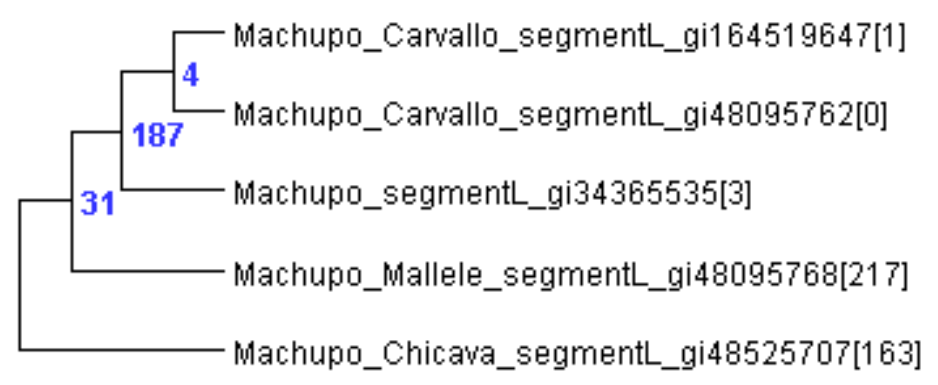


Machupo S segment

Number_SNPs: 614

Number_Homoplastic_SNPs from MSA tree (shown below): 54

Number_Homoplastic_SNPs from SNP Hamming distance tree: 54

Number_Homoplastic_SNPs from SNP RAxML tree: 56

The following 4 strains cannot be uniquely resolved on the basis of SNPs:

1 Machupo_Carvallo_segmentS_gi22901290

1 Machupo_Carvallo_segmentS_gi45826501

1 Machupo_Carvallo_segmentS_gi48095765

1 Machupo_segmentS_gi34365532

Number of SNP microarray probes:

15,309 probes for all alleles

4,336 probes for observed alleles only

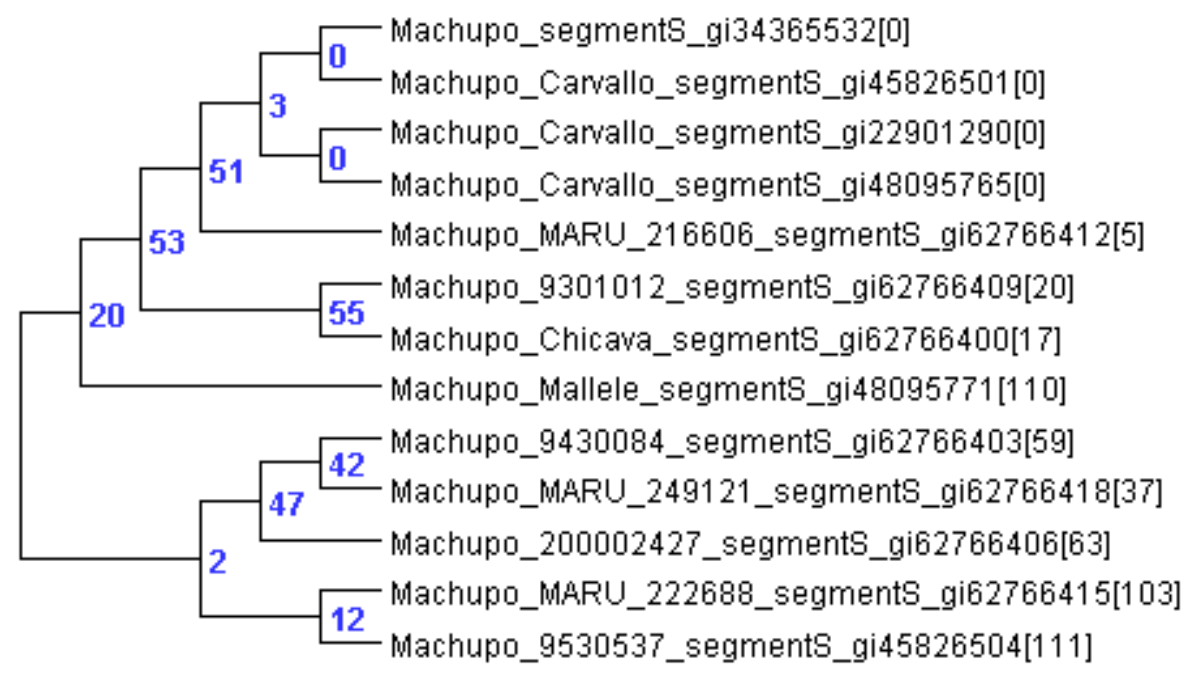


New World Arenaviruses L segment (including Machupo, Junin, and others)

Number_SNPs: 4389

Number_Homoplastic_SNPs from MSA tree: 79

Number_Homoplastic_SNPs from SNP Hamming distance tree (shown below): 50

Number_Homoplastic_SNPs from SNP RAxML tree: 233

All strains can be uniquely resolved on the basis of SNPs.

Number of SNP microarray probes:

80,973 probes for all alleles

20,800 probes for observed alleles only

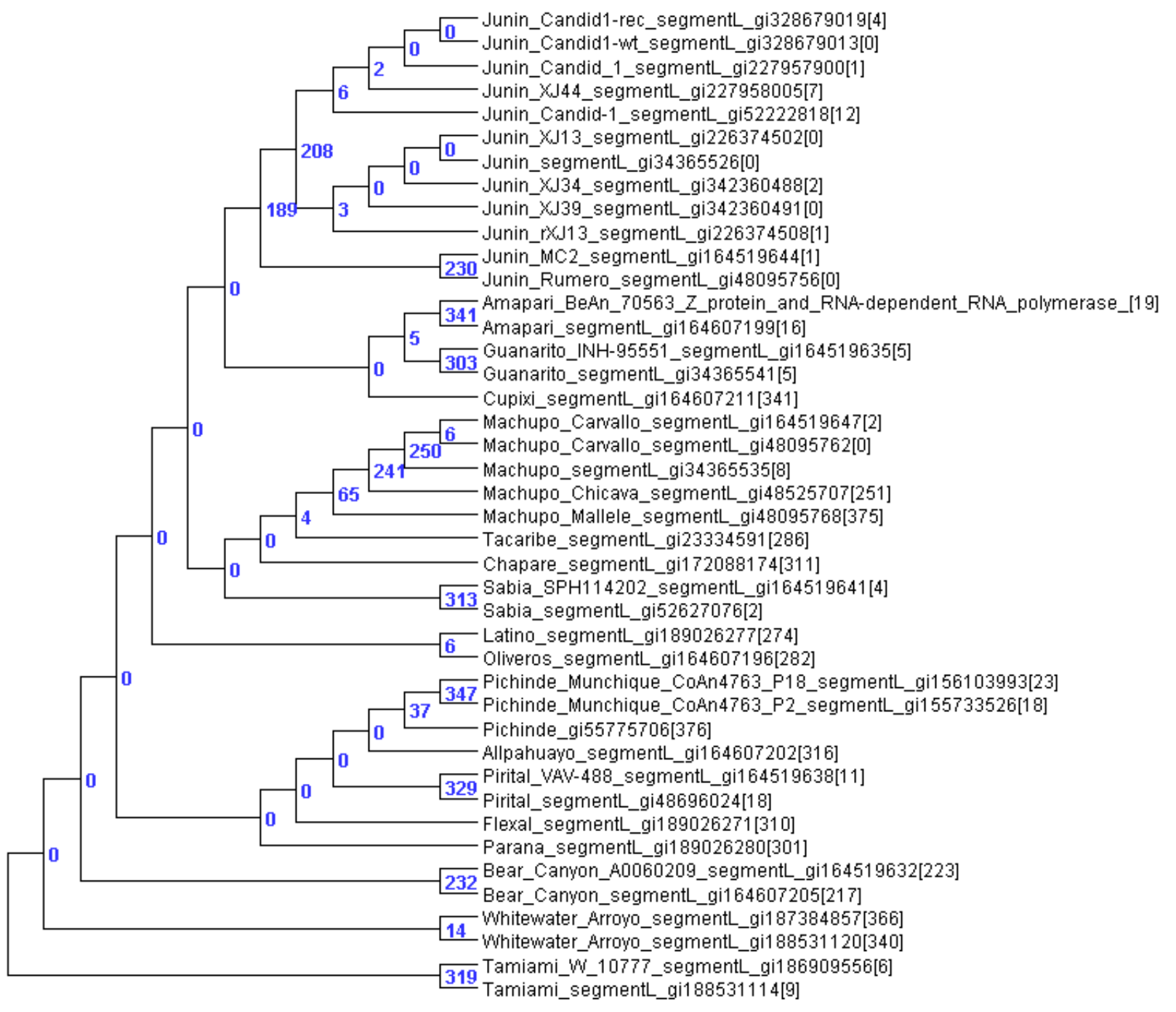


New World Arenaviruses S segment (including Machupo, Junin, and others)

Number_SNPs: 5410

Number_Homoplastic_SNPs from MSA tree: 654

Number_Homoplastic_SNPs from SNP Hamming distance tree (shown below): 521

Number_Homoplastic_SNPs from SNP RAxML tree: 724

The following 2 pairs of strains cannot be uniquely resolved on the basis of SNP:

1 Machupo_Carvallo_segmentS_gi22901290

1 Machupo_Carvallo_segmentS_gi48095765

2 Pichinde_gi332639

2 Pichinde_gi55733698

Number of SNP microarray probes:

139,505 probes for all alleles

37,249 probes for observed alleles only 


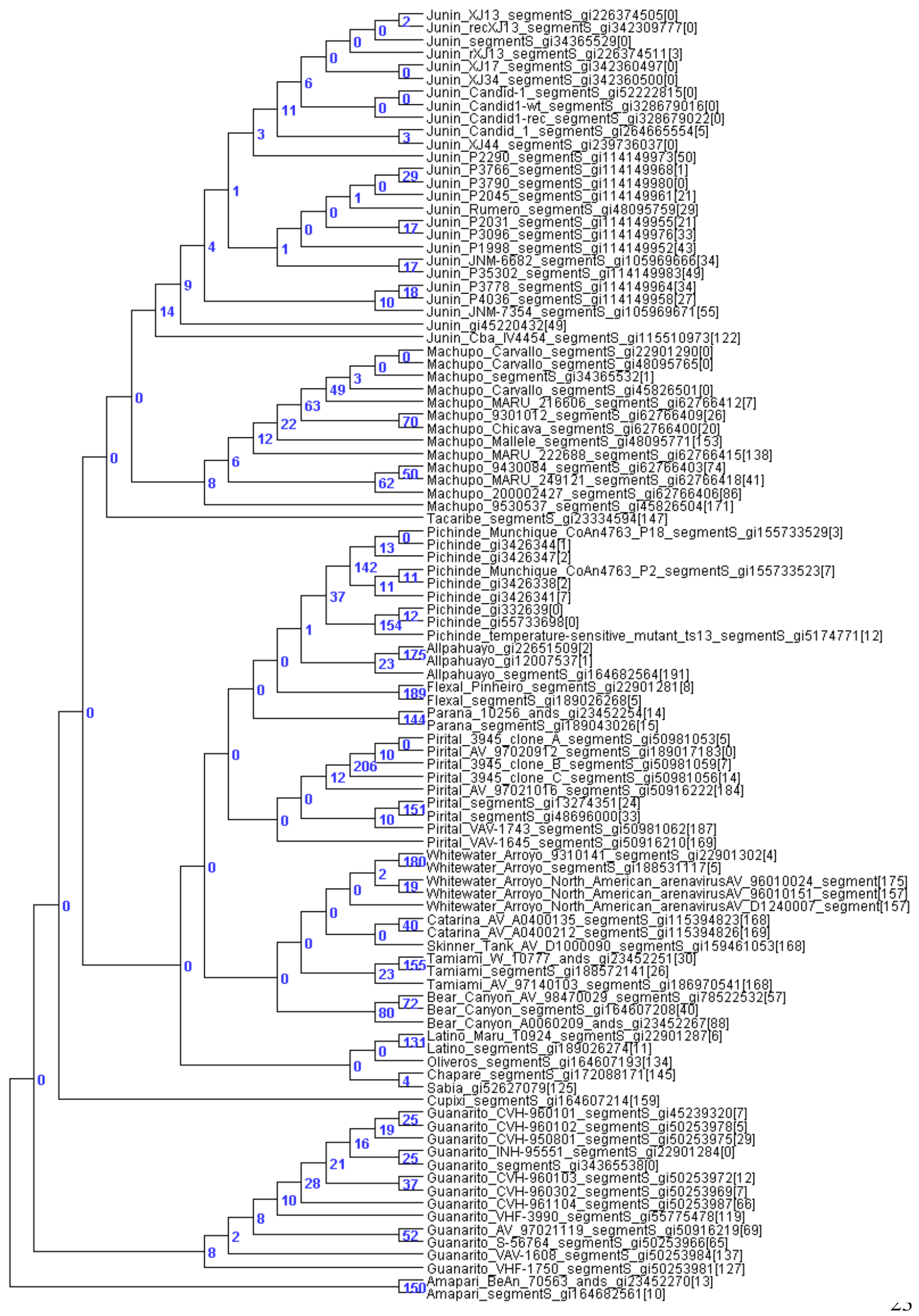


Nipah

Number_SNPs: 684

Number_Homoplastic_SNPs from MSA tree (shown below): 0

Number_Homoplastic_SNPs from SNP Hamming distance tree: 0

Number_Homoplastic_SNPs from SNP RAxML tree: 0

All strains can be uniquely resolved on the basis of SNPs.

Number of SNP microarray probes:

10,573 probes for all alleles

3,090 probes for observed alleles only

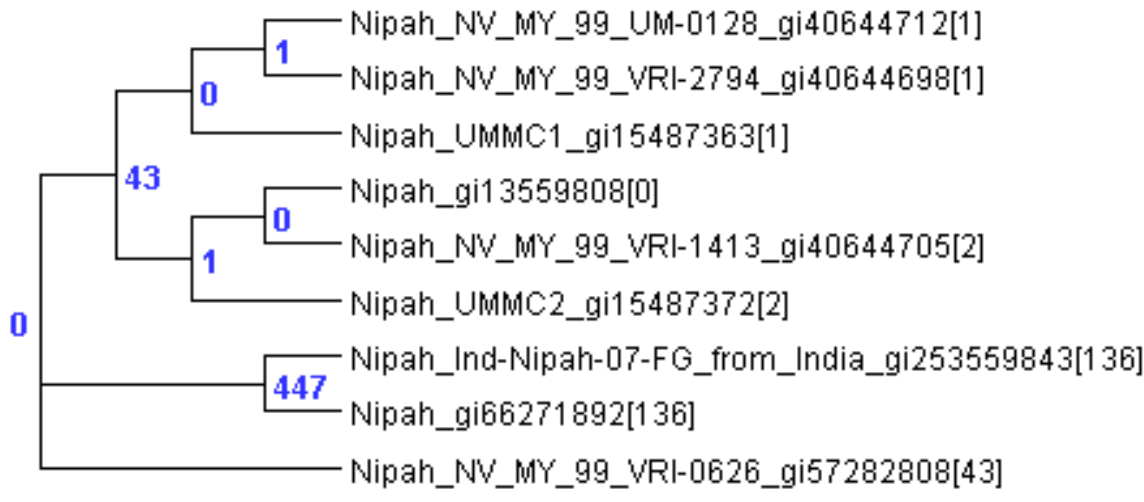




\section{Hendra}

Number_SNPs: 437

Number_Homoplastic_SNPs from MSA tree (shown below): 37

Number_Homoplastic_SNPs from SNP Hamming distance tree: 38

Number_Homoplastic_SNPs from SNP RAxML tree: 37

All strains can be uniquely resolved on the basis of SNPs.

Number of SNP microarray probes:

5,841 probes for all alleles

2,280 probes for observed alleles only

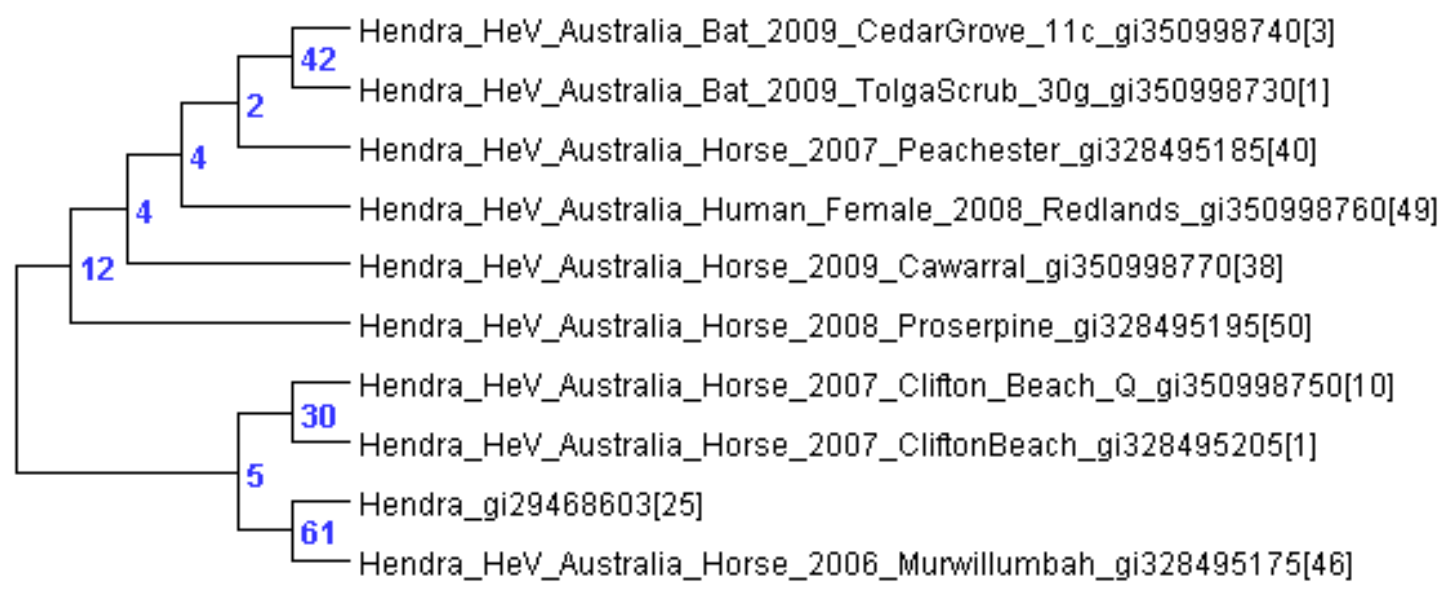


Tick-borne encephalitis virus complex

Number_SNPs: 10,276

Number_Homoplastic_SNPs from MSA tree (shown below): 1260

Number_Homoplastic_SNPs from SNP Hamming distance tree: 1272

Number_Homoplastic_SNPs from SNP RAxML tree: 1759

All strains can be uniquely resolved on the basis of SNPs.

Number of SNP microarray probes:

288,269 probes for all alleles

78,225 probes for observed alleles only 


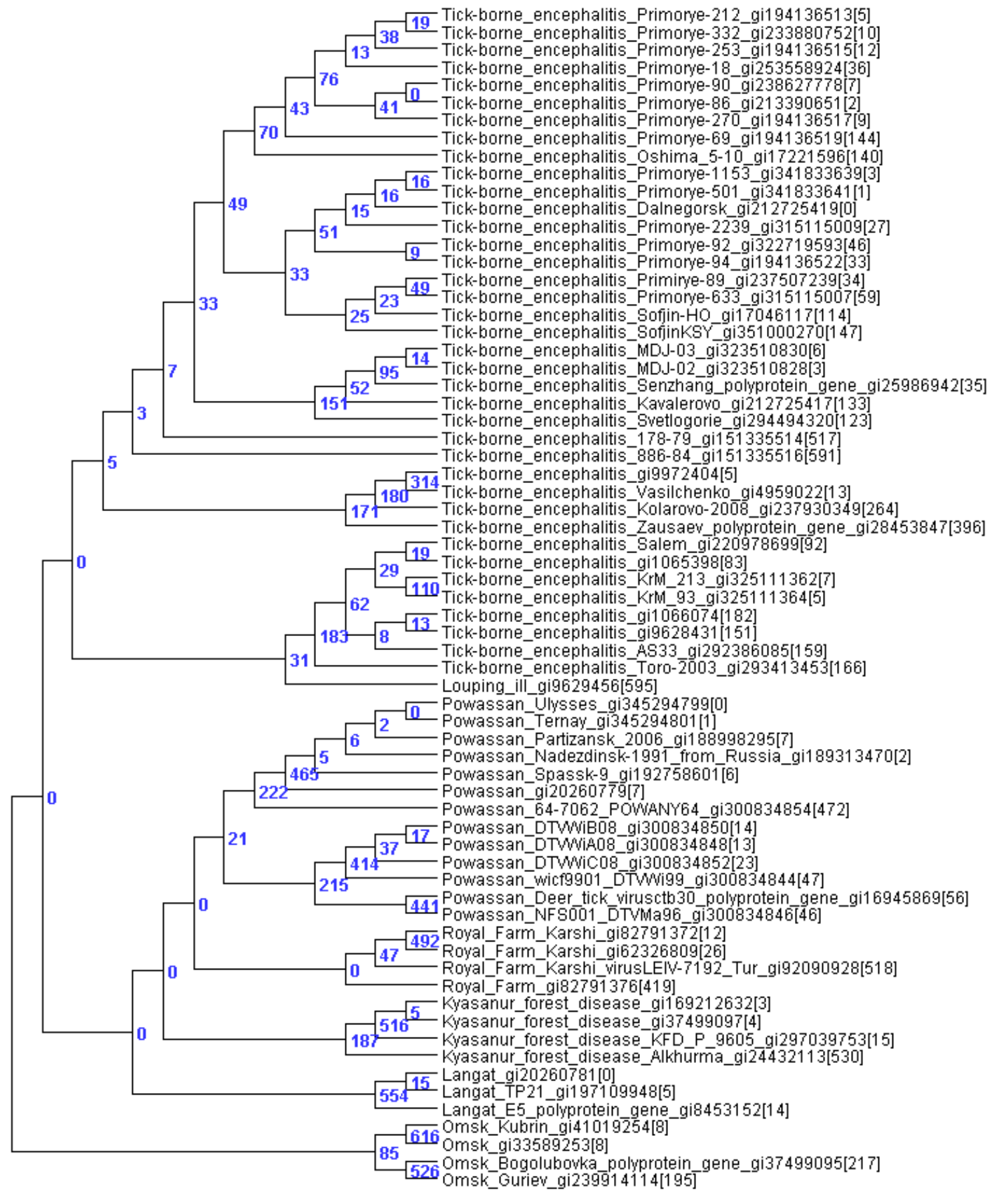




\section{References}

1. Gardner S, Slezak T: Scalable SNP Analyses of 100+ Bacterial or Viral Genomes. $J$ Forensic Res 2010, 1:107, doi:110.4172/2157-7145.1000107.

2. Edgar RC: MUSCLE: multiple sequence alignment with high accuracy and high throughput. Nucleic Acids Res 2004, 32:1792-1797.

3. Stamatakis A: RAxML-VI-HPC: maximum likelihood-based phylogenetic analyses with thousands of taxa and mixed models. Bioinformatics 2006, 22:2688-2690. 